\title{
K-shell photoabsorption and photoionisation of trace elements
}

\section{Isoelectronic sequences with electron number $3 \leq N \leq 11$ (Research Note)}

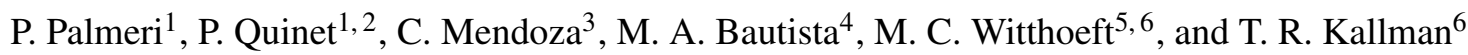 \\ 1 Physique Atomique et Astrophysique, Université de Mons - UMONS, 20 place du Parc, 7000 Mons, Belgium \\ e-mail: patrick.palmeri@umons.ac.be \\ 2 IPNAS, Université de Liège, Campus du Sart Tilman, Bât. B15, 4000 Liège, Belgium \\ e-mail: pascal.quinet@umons.ac.be \\ ${ }^{3}$ Centro de Física, Instituto Venezolano de Investigación Científicas (IVIC), A.P. 21827, Caracas 1020, Venezuela \\ e-mail: claudio@ivic.gov.ve \\ 4 Department of Physics, Western Michigan University, 1903 W Michigan Ave., Kalamazoo, MI 49008, USA \\ 5 ADNET Systems, Inc., Bethesda, MD 20817, USA \\ ${ }^{6}$ NASA Goddard Space Flight Center, Greenbelt, MD 20771, USA
}

Received 8 March 2016 / Accepted 25 March 2016

\begin{abstract}
Context. With the recent launching of the Hitomi X-ray space observatory, $\mathrm{K}$ lines and edges of chemical elements with low cosmic abundances, namely F, Na, P, Cl, K, Sc, Ti, V, Cr, Mn, $\mathrm{Co}, \mathrm{Cu}$ and $\mathrm{Zn}$, can be resolved and used to determine important properties of supernova remnants, galaxy clusters and accreting black holes and neutron stars.

Aims. The second stage of the present ongoing project involves the computation of the accurate photoabsorption and photoionisation cross sections required to interpret the X-ray spectra of such trace elements.

Methods. Depending on target complexity and computer tractability, ground-state cross sections are computed either with the closecoupling Breit-Pauli $R$-matrix method or with the AUTOSTRUCTURE atomic structure code in the isolated-resonance approximation. The intermediate-coupling scheme is used whenever possible. In order to determine a realistic K-edge behaviour for each species, both radiative and Auger dampings are taken into account, the latter being included in the $R$-matrix formalism by means of an optical potential.

Results. Photoabsorption and total and partial photoionisation cross sections are reported for isoelectronic sequences with electron numbers $3 \leq N \leq 11$. The Na sequence $(N=11)$ is used to estimate the contributions from configurations with a 2 s hole (i.e. [2s] $\mu$ ) and those containing $3 \mathrm{~d}$ orbitals, which will be crucial when considering sequences with $N>11$.

Conclusions. It is found that the [2s] $\mu$ configurations must be included in the target representations of species with $N \geq 11$ as they contribute significantly to the monotonic background of the cross section between the $\mathrm{L}$ and $\mathrm{K}$ edges. Configurations with $3 \mathrm{~d}$ orbitals are important in rendering an accurate L edge, but they can be practically neglected in the K-edge region.
\end{abstract}

Key words. atomic data - X-rays: general

\section{Introduction}

$\mathrm{K}$ lines from chemical elements with low cosmic abundances (trace elements) have been observed in the 5-9 keV energy range in spectra taken by the Chandra, XMM-Newton and Suzaku space observatories. Such surprising findings, in spite of trace abundances as low as a factor of 50 relative to $\mathrm{Fe}$, speak for the high sensitivity of the satellite-borne instrumentation but also for puzzling abundance enhancements in some X-ray sources.

$\mathrm{K}$ emission lines from $\mathrm{Cr}$ and $\mathrm{Mn}$, for instance, were first observed in supernova remnants with Chandra (Hwang et al. 2000) and then with Suzaku (Park et al. 2013) showing ionisation levels consistent with those of Fe, thus implying that these lines were emitted in the same spatial region. The $\mathrm{Cr} / \mathrm{Fe}$ abundance ratio has been used by Badenes et al. (2008) as an indicator of progenitor metallicity and of the explosion mechanism. Trace-element lines have also been reported in the spectra of galaxy clusters
(Tamura et al. 2009) and the Galactic centre (Nobukawa et al. 2010).

The spectra of compact X-ray sources, e.g. accreting black holes and neutron stars and their related warm absorbers, display absorption lines and edges - some of which are blue-shifted by gas outflows - that constrain the elemental abundances, ionisation structure, progenitor mass and velocity dispersion. An interesting example is the galactic black hole GRO J1655-40 where, due to line saturation, $\mathrm{K}$ absorption lines from trace elements $(\mathrm{P}$, $\mathrm{K}, \mathrm{Cr}, \mathrm{Mn}$ and $\mathrm{Co}$ ) impose more constrains on the flow properties than those from abundant species such as Fe, S, Si and $\mathrm{Mg}$ (Miller et al. 2006; Kallman et al. 2009). Also in the Chandra HETG spectrum of the galactic black hole GRS1915+105 by Ueda et al. (2009), an unusually broad range of K lines from $\mathrm{Cu}$ ions (with electron numbers $2 \leq N \leq 6$ ) have been identified in what is believed to be a single zone of ionisation equilibrium. 
The Soft X-ray Spectrometer (SXS) aboard the new Hitomi telescope $^{1}$ will offer high sensitivity and spectral resolution $(5 \mathrm{eV})$ in the $5-10 \mathrm{keV}$ spectral range. It will enable the astrophysical exploitation of $\mathrm{K}$ lines and edge structures from trace elements in moderately bright sources with realistic observing times. The SXS energy resolution is expected to be adequate to distinguish between adjacent ionic stages of a $\mathrm{K}$ line or edge.

The quantitative interpretation of X-ray spectra requires the understanding of and reliable data for a whole variety of innershell processes such as K-shell photoabsorption and electron impact excitation, radiative and Auger decay of K-vacancy levels, $\mathrm{K}$-edge resonance structures and $\mathrm{K} \alpha$ fluorescence emission. Although photoionisation cross sections based on central field approximations are currently available for all subshells of most ions of interest (e.g. Verner et al. 1996), they are not accurate enough as they do not take into account resonances and damping (radiative and Auger) effects that dominate the K-edge behaviour (edge smearing) and location.

As the first stage of a project to compute the required innershell data for the spectral modelling of trace elements in X-ray sources, Palmeri et al. (2012, hereafter Paper I) have recently reported - for the complete isonuclear sequences of F, Na, P, $\mathrm{Cl}, \mathrm{K}, \mathrm{Sc}, \mathrm{Ti}, \mathrm{V}, \mathrm{Cr}, \mathrm{Mn}, \mathrm{Co}, \mathrm{Cu}$ and $\mathrm{Zn}-$ energies, radiative and Auger widths and fluorescence yields for K-vacancy levels; and wavelengths and radiative $A$-values for their transitions to valence levels. These data were mainly calculated with the Hartree-Fock method with relativistic corrections (HFR) of Cowan (1981), but data accuracy was assessed with comparisons with the multiconfiguration Breit-Pauli (AUTOSTRUCTURE, Eissner et al. 1974; Badnell 2011) and the multiconfiguration Dirac-Fock (GRASP, Grant et al. 1980; McKenzie et al. 1980) methods.

As a followup, we are currently involved in the extensive computations of the ground-state photoabsorption and photoionisation cross sections of the all the charge states of the aforementioned isonuclear sequences. In this paper we delineate the strategy and methods to accomplish this task, and report cross sections for the simpler systems with electron numbers $3 \leq N \leq 11$. We conveniently draw from our earlier work on K photoabsorption of cosmic abundant species (Palmeri et al. 2002, 2005; Bautista et al. 2004; García et al. 2005, 2009; Witthoeft et al. 2009，2011a,b, 2013; Gorczyca et al. 2013; Hasoğlu et al. 2014). The final data sets of the present project are destined to improve the atomic database of the XSTAR photoionisation modelling code (Bautista \& Kallman 2001).

\section{Numerical methods}

The main problem in rendering the inner-shell photoabsorption and photoionisation cross sections of the ionic systems considered in the present project is computer tractability, as target representations with electron number $N>11$ quickly lead to unmanageably large close-coupling calculations. Consequently, some compromises have to be made within the context of the multichannel approach (e.g. $L S$ coupling vs. intermediate coupling), or altogether downsize, in spite of its shortcomings (Badnell et al. 1993), to the isolated-resonance approximation as extensively implemented to treat inner-shell opacity contributions in the Opacity Project (Badnell et al. 2005). These issues have been previously discussed by Witthoeft et al. (2009, $2011 a, b)$, and therefore they will only be briefly reviewed here.

1 http://heasarc.gsfc.nasa.gov/docs/astroh/
In the close-coupling framework, photoabsorption and photoionisation cross sections are computed with the relativistic (Breit-Pauli) $R$-matrix method (Berrington et al. 1995), using the RMATRX1 package in the inner region and STGBF0DAMP7 in the asymptotic region. The latter allows through an optical potential (Gorczyca \& Badnell 1996, 2000) for the treatment of spectatorAuger damping effects, which are dominant in the near K-edge region. In the isolated-resonance approximation, the cross sections are calculated with the Breit-Pauli atomic structure code AUTOSTRUCTURE (Eissner et al. 1974; Badnell 2011).

For each isonuclear series (identified with the atomic number $Z$ ), photoabsorption and total and partial photoionisation cross sections are computed for members with $3 \leq N \leq Z$ (the difficult near-neutral members with $Z-1 \leq N \leq Z$ will be considered in a more advanced project). We adopt the following computational strategy along isoelectronic sequences.

- $3 \leq N \leq 11$. Calculations are performed in intermediate coupling with RMATRX1 using the target representations (level energies and Auger widths) listed in Table 9 of Paper I. Target wave functions are computed with the AUTOSTRUCTURE code.

- $12 \leq N \leq 20$. Depending on computer tractability, calculations are performed in intermediate coupling with RMATRX1; otherwise $L S$ coupling is implemented but including the non-fine-structure mass-velocity and Darwin interactions. Regarding target representations, two issues are hereby examined with respect to the atomic models tabulated in Paper I: the impact on the background cross section and edge structures due the exclusion of configurations with a $2 \mathrm{~s}$ hole, i.e. [2s] $\mu$ where $\mu$ stands for all occupied valence subshells, and those containing $3 \mathrm{~d}$ orbitals.

- $21 \leq N \leq Z-2$. Whenever possible, RMATRX1 will be used otherwise we will recur to the isolated-resonance scheme with AUTOSTRUCTURE.

\section{Results}

Photoabsorption and total and partial (leaving the daughter ion in the ground and excited levels) photoionisation cross sections have been computed for the ground state and groundconfiguration metastable states of ions of $\mathrm{F}, \mathrm{Na}, \mathrm{P}, \mathrm{Cl}, \mathrm{K}, \mathrm{Sc}, \mathrm{Ti}$, $\mathrm{V}, \mathrm{Cr}, \mathrm{Mn}, \mathrm{Co}, \mathrm{Cu}$ and $\mathrm{Zn}$ with electron numbers $3 \leq N \leq 11$. Target representations (level energies and Auger widths) are taken from Table 9 of Paper I. Although we are mainly interested in the K-edge region, due to the general absence of previous intermediate-coupling multichannel cross sections for these species, we have made an effort to also resolve the L resonances; thus, the cross-section energy tabulations start right from the reaction threshold with a fine mesh step of $E / z^{2}=0.0001 \mathrm{Ryd}$ where $z=Z-N+1$.

We plot in Fig. 1 the K-edge-region photoabsorption cross section of the $2 \mathrm{p}^{6}{ }^{1} \mathrm{~S}_{0}$ ground state of different members of the $\mathrm{Ne}$ isoelectronic sequence. Cross sections have been convolved with a Gaussian of width $\Delta E / E=0.001$ comparable to the SXS spectral resolution of $5 \mathrm{eV}(0.4 \mathrm{Ryd})$. It may be seen that the resonance energy intervals in the K-edge structure increase with $Z$, but even at low $Z$ and this resolution, the $\mathrm{K} \beta$ line can be resolved from the edge structure. This point is further illustrated in Fig. 2 where the closely spaced K lines for phosphorus ions (i.e. systems with a fairly low $Z$ ) with electron numbers $7 \leq N \leq 10$ are shown, the cross sections being again convolved with a Gaussian of the same width. It may be appreciated that the energy intervals in between the $\mathrm{K} \alpha$ lines from the adjacent 


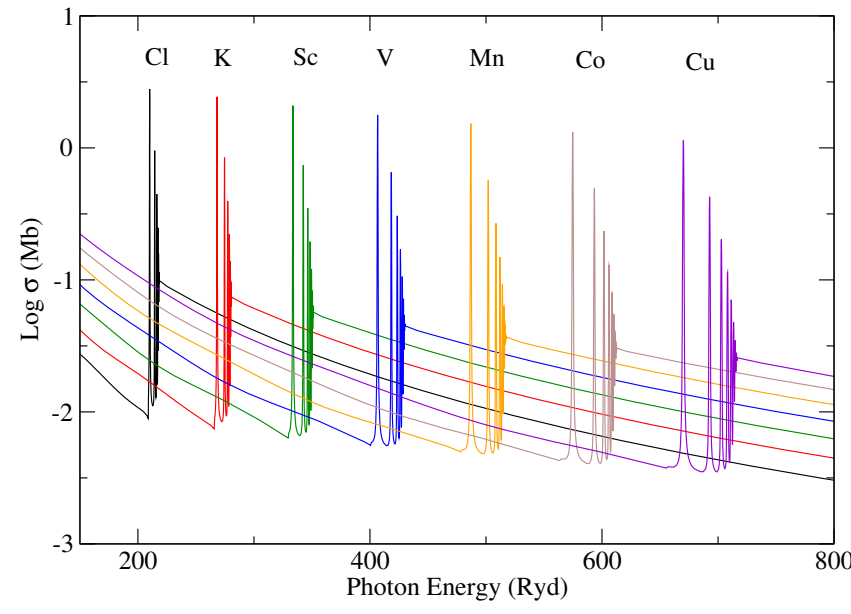

Fig. 1. Photoabsorption cross section in the K-edge region of the $2 \mathrm{p}^{6}{ }^{1} \mathrm{~S}_{0}$ ground state of members of the $\mathrm{Ne}$ isoelectronic sequence. The cross sections have been convolved with a Gaussian of width $\Delta E / E=0.001$.

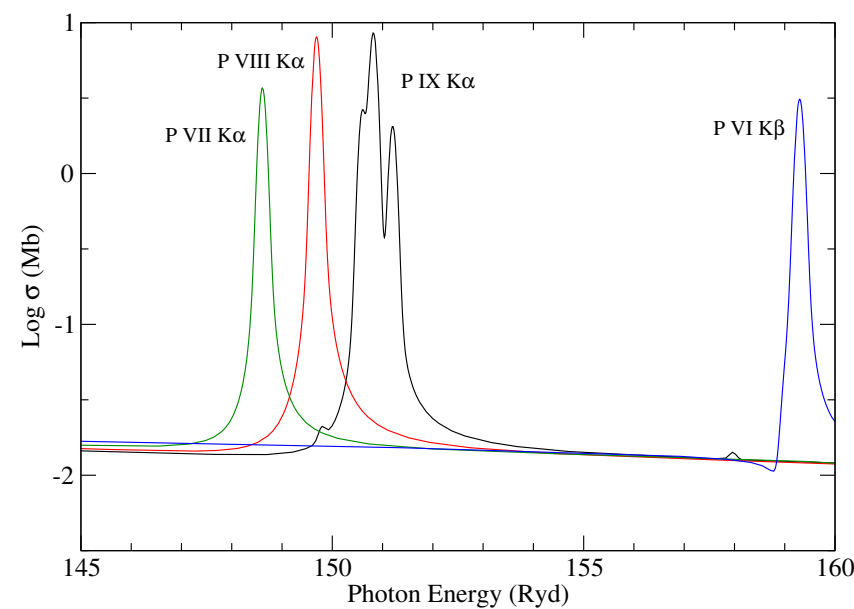

Fig. 2. Photoabsorption cross section in the K-edge region of the ground state of four P ions. Black curve: PIX (N-like). Red curve: P VIII (Olike). Green curve: P VII (F-like). Blue curve: P VI (Ne-like). The cross sections have been convolved with a Gaussian of width $\Delta E / E=0.001$.

$\mathrm{N}$-, O- and F-like ions and the $\mathrm{K} \beta$ line from the Ne-like ion are larger than 0.4 Ryd, and can therefore be resolved by the SXS.

In the ion representations listed in Table 9 of Paper I for isoelectronic sequences with $N \geq 10$, the [2s] $\mu$ configurations and those containing $3 \mathrm{~d}$ orbitals have been excluded. If these target representations are adequate in the present photoionisation computations, it would open the possibility of using the Breit-Pauli $R$-matrix method in intermediate coupling up to at least $N \leq 20$. Therefore, the contributions from these configurations must be evaluated before they are discarded altogether, if at all, and the relatively simple $\mathrm{Na}$ isoelectronic sequence presents a convenient way to carry out these tests. We thus consider the following three Ne-like target approximations.

Target $A$ : it is represented by the configurations $2 p^{6},[2 p] 3 s$, $[2 \mathrm{p}] 3 \mathrm{p},[1 \mathrm{~s}] 3 \mathrm{~s}$ and $[1 \mathrm{~s}] 3 \mathrm{p}$.

Target $B$ : it includes the configurations of Target A plus $[2 \mathrm{~s}] 3 \mathrm{~s}$ and $[2 \mathrm{~s}] 3 \mathrm{p}$.

Target $C$ : it includes the configurations of Target B plus $[2 \mathrm{p}] 3 \mathrm{~d}$, $[2 \mathrm{~s}] 3 \mathrm{~d}$ and $[1 \mathrm{~s}] 3 \mathrm{~d}$.

Target A is the one listed in Table 9 of Paper I while Target B additionally includes the $[2 \mathrm{~s}] 3 \mathrm{~s}$ and $[2 \mathrm{~s}] 3 \mathrm{p}$ configurations with
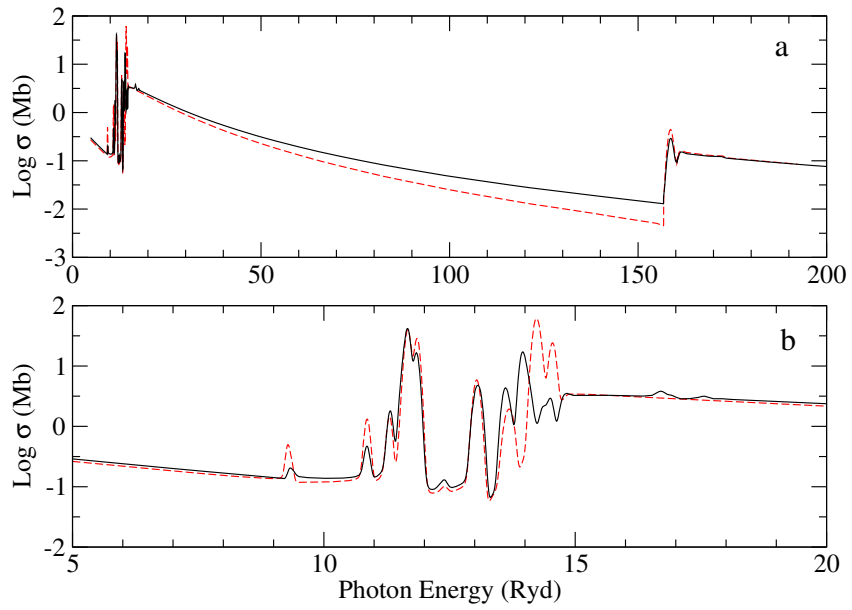

Fig. 3. Photoabsorption cross section of the $2 p^{6} 3 s^{2} S_{1 / 2}$ ground state of Na-like P V computed with Target B (black continuous curve) and Target A (red dashed curve). a) Energy region between the L and $\mathrm{K}$ edges. b) L-edge region. The cross sections have been convolved with a Gaussian of width $\Delta E / E=0.01$.

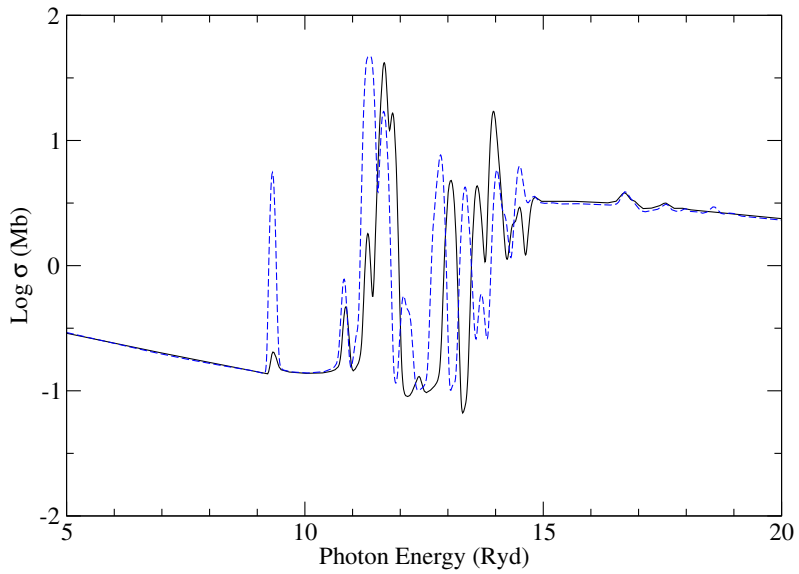

Fig. 4. Photoabsorption cross sections of the $2 p^{6} 3 s^{2} S_{1 / 2}$ ground state of $\mathrm{Na}$-like $\mathrm{P} \mathrm{V}$ in the L-edge region computed with Target $\mathrm{B}$ (black continuous curve) and Target $C$ (blue dashed curve). The cross sections have been convolved with a Gaussian of width $\Delta E / E=0.01$.

a 2 s hole. Target $\mathrm{C}$ takes all the possible inner-shell single promotions to the $3 \ell$ orbitals with $\ell \leq 2$. In Fig. 3 the photoabsorption cross sections of the $2 \mathrm{p}^{6} 3 \mathrm{~s}{ }^{2} \mathrm{~S}_{1 / 2}$ ground state of $\mathrm{PV}$ (Na-like) computed with Target A and Target B are depicted. In order to bring out the main features, the cross sections are convolved with a low resolution $(\Delta E / E=0.01)$ Gaussian profile. It may be appreciated in Fig. 3a that, while the structure of the $\mathrm{K}$ edge is similar between the two approximations, Target $\mathrm{B}$ gives a significantly lower gradient in the monotonic decrease of the cross section between the L ( $\sim 15 \mathrm{Ryd})$ and the K ( $\sim 160 \mathrm{Ryd})$ edges. As shown in Fig. 3b, there are also some noticeable differences in the L-edge resonance structure around the spectral head at $\sim 14-15$ Ryd. On the other hand, it is found that Target C leads to conspicuous differences with respect to Target B only in the L-edge resonance structure as shown in Fig. 4, where it may be seen that there are significant shifts in the resonance positions and disimilar resonance profile characteristics (width and height); however, we find that an acceptable level of convergence has been attained for this system with Target $\mathrm{C}$ (single promotions). 


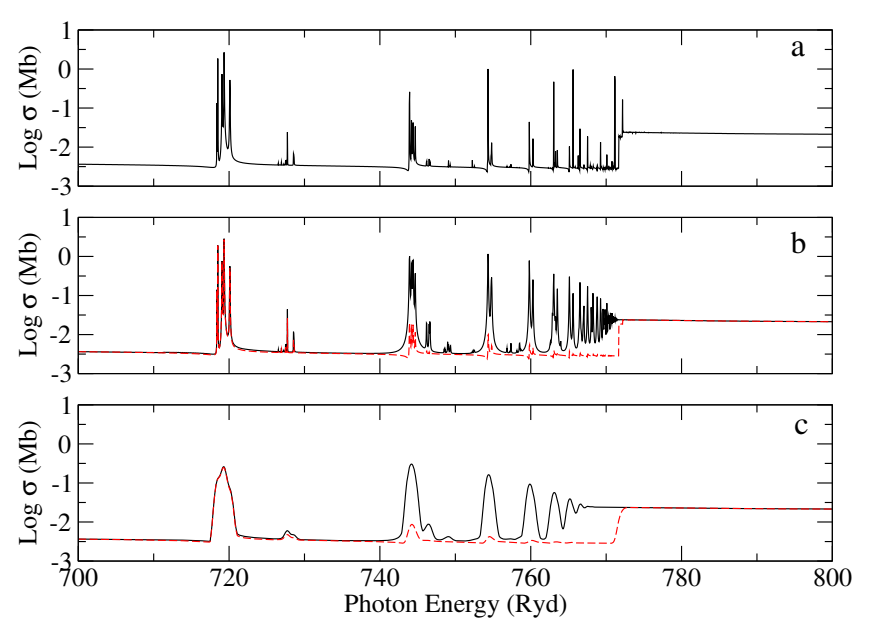

Fig. 5. Photoabsorption (black continuous curve) and photoionisation (red dashed curve) of the $2 \mathrm{p}^{6} 3 \mathrm{~s}^{2} \mathrm{~S}_{1 / 2}$ ground state of Na-like $\mathrm{Zn} \mathrm{XX}$. a) Undamped cross section. b) Damped cross sections. c) Damped cross sections convolved with a Gaussian of width $\Delta E / E=0.001$.

The photoabsorption and photoionizaton cross sections of the $2 \mathrm{p}^{6} 3 \mathrm{~s}{ }^{2} \mathrm{~S}_{1 / 2}$ ground state of Na-like $\mathrm{ZnXX}$ are plotted in Fig. 5 to illustrate the importance of damping effects. In Fig. 5a damping is excluded and, therefore, the photoabsorption and photoionisation cross sections are alike. Damping is taken into account in Fig. 5b, and as a consequence, the resonances in the photoabsorption cross section show symmetric profiles of constant widths that causes the smearing of the $\mathrm{K}$ edge; however, the $\mathrm{K}$ edge remains sharp in the photoionisation cross section although most of the resonances are distinctively damped. As shown in Fig. 5c, the position and smearing of the $\mathrm{K}$ edge in the photoabsorption cross section depends on the convolution width, in this case a Gaussian of width $\Delta E / E=0.001$; moreover, the $\mathrm{K}$ edge in the photoionisation cross section is now slightly slanted.

\section{Conclusions}

1. Photoabsorption and photoionisation (total and partial) cross sections for the ions of F, Na, P, Cl, K, Sc, Ti, V, Cr, Mn, Co, $\mathrm{Cu}$ and $\mathrm{Zn}$ with electron numbers $3 \leq N \leq 11$ have been computed with the Breit-Pauli $R$-matrix method in intermediate coupling. Both the $\mathrm{L}$ and $\mathrm{K}$ resonance structures are well resolved with a fine energy mesh. Damping effects are conspicuous in the K-edge resonance structure.

2. It has been shown that, with the SXS nominal resolution of $\Delta E=5 \mathrm{eV}(0.4 \mathrm{Ryd})$, it is possible to resolve the $\mathrm{K} \alpha$ and $\mathrm{K} \beta$ of these trace elements, even those with low $Z$ (e.g. $Z \sim 15$ ).

3. Even though the ion representations listed in Paper I are adequate targets for isoelectronic sequences with $N \leq 10$, it has been shown here that for $N>10$ the [2s] $\mu$ configurations, which were not considered in Paper I, must be additionally included in the close-coupling expansion as they lead to significant contributions to the background cross section in the energy region between the $\mathrm{L}$ and $\mathrm{K}$ edges.

4. The inclusion of $3 \mathrm{~d}$ configurations in the target representations of Na-like ions $(N=11)$ mainly leads to noticeable contributions in the resonance structure of the L edge, but the $\mathrm{K}$ edge and the background cross sections are hardly modified. However, they certainly lead to strong configuration interaction among $n=3$ valence states, but calculations are expected to grow very rapidly with $N$ making intermediatecoupling $R$-matrix calculations very large. This implies that the photoionisation cross sections in the L- and K-edge regions of ions with $N>11$ will most surely have to be computed separately with different target approximations.

5. Although cross sections for particular species can be obtained directly from the authors, they will only be published as a whole when the data sets are eventually completed. This will avoid data duplication in case there is need to rerun any isoelectronic sequence.

Acknowledgements. This project is sponsored by the NASA grant 12-APRA120070 through the Astrophysics Research and Analysis Program.

\section{References}

Badenes, C., Hughes, J. P., Cassam-Chenaï, G., \& Bravo, E. 2008, ApJ, 680, 1149

Badnell, N. R. 2011, Comp. Phys. Comm., 182, 1528

Badnell, N. R., Griffin, D. C., Gorczyca, T. W., \& Pindzola, M. S. 1993, Phys. Rev. A, 48, 2519

Badnell, N. R., Bautista, M. A., Butler, K., et al. 2005, MNRAS, 360, 458

Bautista, M. A., \& Kallman, T. R. 2001, ApJS, 134, 139

Bautista, M. A., Mendoza, C., Kallman, T. R., \& Palmeri, P. 2004, A\&A, 418, 1171

Berrington, K. A., Eissner, W. B., \& Norrington, P. H. 1995, Comp. Phys. Comm., 92, 290

Cowan, R. D. 1981, The theory of atomic structure and spectra (Berkley: University of California Press)

Eissner, W., Jones, M., \& Nussbaumer, H. 1974, Comp. Phys. Comm., 8, 270

García, J., Mendoza, C., Bautista, M. A., et al. 2005, ApJS, 158, 68

García, J., Kallman, T. R., Witthoeft, M., et al. 2009, ApJS, 185, 477

Gorczyca, T. W., \& Badnell, N. R. 1996, J. Phys. B Atom. Mol. Phys., 29, L283 Gorczyca, T. W., \& Badnell, N. R. 2000, J. Phys. B Atom. Mol. Phys., 33, 2511 Gorczyca, T. W., Bautista, M. A., Hasoglu, M. F., et al. 2013, ApJ, 779, 78

Grant, I. P., McKenzie, B. J., Norrington, P. H., Mayers, D. F., \& Pyper, N. C. 1980, Comp. Phys. Comm., 21, 207

Hasoğlu, M. F., Abdel-Naby, S. A., Gatuzz, E., et al. 2014, ApJS, 214, 8

Hwang, U., Petre, R., \& Hughes, J. P. 2000, ApJ, 532, 970

Kallman, T. R., Bautista, M. A., Goriely, S., et al. 2009, ApJ, 701, 865

McKenzie, B. J., Grant, I. P., \& Norrington, P. H. 1980, Comp. Phys. Comm., 21, 233

Miller, J. M., Raymond, J., Fabian, A., et al. 2006, Nature, 441, 953

Nobukawa, M., Koyama, K., Tsuru, T. G., Ryu, S. G., \& Tatischeff, V. 2010, PASJ, 62, 423

Palmeri, P., Mendoza, C., Kallman, T. R., \& Bautista, M. A. 2002, ApJ, 577, L119

Palmeri, P., Kallman, T. R., Mendoza, C., Bautista, M. A., \& Krolik, J. H. 2005, in X-ray Diagnostics of Astrophysical Plasmas: Theory, Experiment, and Observation, ed. R. Smith, AIP Conf. Ser., 774, 75

Palmeri, P., Quinet, P., Mendoza, C., et al. 2012, A\&A, 543, A44

Park, S., Badenes, C., Mori, K., et al. 2013, ApJ, 767, L10

Tamura, T., Maeda, Y., Mitsuda, K., et al. 2009, ApJ, 705, L62

Ueda, Y., Yamaoka, K., \& Remillard, R. 2009, ApJ, 695, 888

Verner, D. A., Ferland, G. J., Korista, K. T., \& Yakovlev, D. G. 1996, ApJ, 465, 487

Witthoeft, M. C., Bautista, M. A., Mendoza, C., et al. 2009, ApJS, 182, 127

Witthoeft, M. C., Bautista, M. A., García, J., et al. 2011a, ApJS, 196, 7

Witthoeft, M. C., García, J., Kallman, T. R., et al. 2011b, ApJS, 192, 7

Witthoeft, M. C., Bautista, M. A., García, J., et al. 2013, Atom. Data Nucl. Data, 99,53 